\title{
Validade de construto do Functional Impairment Test-Hand and Neck/Shoulder and Arm (FIT-HANSA) para trabalhadores com queixas no membro superior de um hospital terciário
}

\section{Construct validity of the Functional Impairment Test-Hand and Neck/Shoulder and Arm (FIT-HANSA) for workers with upper limb complaints of a tertiary hospital}

(D) Natália Claro da Silva1, (D Lívia Nahas Pinola ${ }^{1}$, (D) Flávia Pessoni Faleiros Macêdo Ricci ${ }^{1}$, (D) Thais Marques Fifolato ${ }^{1}$, (D) Ester Rodrigues do Carmo Lopes², ${ }^{2}$ Karen Ayumi Kawano Suzuki², (D) Marisa de Cássia Registro Fonseca ${ }^{3}$

\begin{abstract}
RESUMO
O Functional Impairment Test-Hand and Neck/Shoulder/Arm (FIT-HaNSA) é um instrumento que avalia a resistência à fadiga e o desempenho do membro superior por meio de funções motoras grossas comumente utilizadas no dia a dia. Objetivo: Analisar a validade de construto do FIT-HaNSA em trabalhadores com sintomas no membro superior, por meio da correlação entre a resistência à fadiga do segmento com os escores de força muscular do ombro, cotovelo e mão, com a capacidade para o trabalho (ICT) e com a disfunção do membro superior (QUICK DASH-Br). Métodos: Trinta e nove trabalhadores de um hospital terciário com idade média de 42,9 anos (DP13,29) foram recrutados. Os instrumentos de medida Isocinético Biodex System $4 \mathrm{Pro}^{\mathrm{TM}}$, dinamômetro de preensão palmar JAMAR ${ }^{\circledR}$, QuickDASH-Br e ICT foram aplicados e correlacionados com o FIT-HaNSA. O Coeficiente de Correlação de Pearson ( $r$ ) foi utilizado para quantificar a associação entre os instrumentos. Resultados: O FIT-HaNSA apresentou de fraca a moderada correlação entre a força muscular do ombro, cotovelo e mão $(r=0,18-0,58)$, porém significativas para ombro e mão. A associação entre o FIT-HaNSA com o QuickDASH-Br e ICT se mostraram fracas ( $r=-0,38$ e 0,21$)$. Conclusão: Nosso estudo forneceu evidências preliminares com relação às relações esperadas e validade do FIT-HaNSA como instrumento de medida para a avaliação da fadiga e desconforto em trabalhadores com queixas no membro superior.
\end{abstract}

Palavras-chave: Transtornos Traumáticos Cumulativos, Fadiga Muscular, Extremidade Superior

${ }^{1}$ Fisioterapeuta, Pós-Graduação em Reabilitação e Desempenho Funcional, Faculdade de Medicina de Ribeirão Preto, Universidade de São Paulo

2 Fisioterapeuta, Faculdade de Medicina de Ribeirão Preto, Universidade de São Paulo

3 Fisioterapeuta, Centro de Reabilitação do Hospital das Clínicas de Ribeirão Preto, Faculdade de Medicina de Ribeirão Preto, Universidade de São Paulo

Correspondência

Natália Claro da Silva

E-mail: natalia.claro@hotmail.com

Submetido: 27 Outubro 2020

Aceito: 5 Novembro 2020

Como citar

Silva NC, Pinola LN, Ricci FPFM, Fifolato TM, Lopes ERC, Suzuki KAK, et al. Validade de construto do Functional Impairment Test-Hand and Neck/Shoulder and Arm (FIT-HANSA) para trabalhadores com queixas no membro superio de um hospital terciário. Acta Fisiatr. 2020;27(3):182-189.

DOI: 10.11606/issn.2317-0190.v27i3a176784

\section{ABSTRACT}

The Functional Impairment Test-Hand and Neck/Shoulder/Arm (FIT-HaNSA) is an instrument that assesses fatigue resistance and upper limb performance through thick motor functions commonly used in everyday life. Objective: To analyze the construct validity of the FIT-HaNSA in workers with symptoms in the upper limb, through the correlation between the fatigue resistance of the segment with the muscle strength scores of the shoulder, elbow and hand, with the ability to work (ICT) and with upper limb dysfunction (QUICK DASH-Br). Methods: Thirty-nine workers from a tertiary hospital with an average age of 42.9 years (SD13.29) were recruited. The Biodex System 4 Pro $^{\mathrm{TM}}$ isokinetic measurement instruments, JAMAR ${ }^{\circledR}$ handgrip dynamometer, QuickDASH-Br and ICT were applied and correlated with FIT-HaNSA. Pearson's correlation coefficient $(r)$ was used to quantify the association between the instruments. Results: FIT-HaNSA showed a weak to moderate correlation between the muscular strength of the shoulder, elbow and hand $(r=$ $0.18-0.58)$, but significant for the shoulder and hand. The association between FIT-HaNSA with QuickDASH-Br and ICT was weak ( $r=-0.38$ and 0.21 ). Conclusion: Our study provided preliminary evidence regarding the expected relationships and validity of FIT-HaNSA as a measurement tool for the assessment of fatigue and discomfort in workers with complaints in the upper limb.

Keywords: Cumulative Trauma Disorders, Muscle Fatigue, Upper Extremity, Validation Study 


\section{INTRODUÇÃO}

O membro superior é um dos segmentos mais importantes do corpo, sendo responsável e dotado de capacidade funcional para realizar as atividades de vida diária, autocuidado, hobbies e atividades esportivas. ${ }^{1}$ Portanto, as habilidades funcionais, desempenho no trabalho e a qualidade de vida são prejudicados na presença de dor e falta de mobilidade do segmento. ${ }^{2}$

A força no membro superior está diretamente relacionada com a funcionalidade e qualidade de vida, já a fraqueza muscular dificulta a realização de atividades básicas como alimentação e autocuidado. ${ }^{3}$ Sendo assim, a força muscular é considerada um importante preditor de função, mobilidade, independência e atividades de vida diária. ${ }^{4,5}$

As demandas físicas envolvendo trabalho repetitivo, falta de recuperação muscular, precisão de movimentos e posturas incômodas são fatores de risco para o desenvolvimento de disfunções musculoesqueléticas dos membros superiores. ${ }^{6,7}$

O declínio da capacidade dos músculos em gerar força e potência durante uma tarefa física é conhecido como fadiga muscular, também caracterizada por qualquer perda no desempenho total não induzida por exercício devido a vários fatores fisiológicos ou psicológicos. ${ }^{8}$ Quando o membro superior se encontra em estado de fadiga e dor, disfunções musculoesqueléticas são frequentes e estas podem ocasionar a diminuição da produtividade e falta de qualidade de vida, que a longo prazo podem refletir em distúrbios osteomusculares relacionados ao trabalho (DORTs)..$^{9}$ A ocorrência das DORTs constitui um problema crescente no mundo em desenvolvimento, com alta prevalência, gera frequentemente diminuição do rendimento no posto de trabalho, absenteísmo e consequências econômicas. .,10,11 $^{6}$

Dada a importância do membro superior, informações mais precisas sobre as demandas de trabalho relacionadas a este segmento são fundamentais e podem ser obtidas por meio de uma avaliação adequada e direcionada às necessidades do indivíduo. ${ }^{12}$ Deve ser realizado um exame musculoesquelético criterioso que abranja todas as variáveis associadas à saúde do indivíduo, que incluem os sinais e sintomas, nível de atividade e função, fadiga e força, além de dados coletados a partir do autorrelato. ${ }^{13}$

As medidas autorreferidas das funções dos membros superiores são importantes para traduzir o impacto da condição na saúde do paciente e para determinar as mudanças clínicas importantes de caráter relevante para o mesmo. ${ }^{14,15}$

A maioria dos testes de fadiga muscular utilizados atualmente são de autorrelato, no entanto, a linguagem, cultura, comprometimento cognitivo, depressão, resposta afetiva a doenças, educação, fatores ambientais e fatores pessoais podem influenciar a autopercepção. ${ }^{16}$

Dentre os questionários de autorrelato disponíveis para a avaliação do membro superior e demandas de trabalho, podemos citar o Disability of the Arm, Shoulder and Hand $\mathrm{DASH}^{17}$ e sua versão reduzida o Quick-DASH-Br, ${ }^{18}$ o Índice de Capacidade para o Trabalho (ICT), ${ }^{19}$ o Upper Limb Functional Index (ULFI) ${ }^{20}$ e o Michigan Outcome Questionnaire. ${ }^{21}$

No que diz respeito à avaliação da fadiga muscular, muitas escalas já foram desenvolvidas tais como, a Upper Extremity
Work Demands Scale (UEWD), ${ }^{22}$ Strain Index, Rapid Upper-Limb Assessment, ${ }^{12}$ Escala de avaliação de Fadiga (EAF), ${ }^{23}$ e também os testes de resistência a fadiga e desempenho funcional do membro superior tais como o Upper Quarter Y-Balance Test (YBT-UQ), ${ }^{24}$ o Closed kinetic chain upper extremity stability test $(C K C-U E S T)^{25}$ e o Functional Impairment Test-Hand and Neck/Shoulder/Arm (FIT-HaNSA), ${ }^{26}$ além dos dados de resistência a fadiga que podem ser extraídos do dinamômetro isocinético.

Para que este tipo de instrumento seja aplicado e obtenha informações fidedignas, a análise de suas propriedades psicométricas se faz necessária para determinar a qualidade da ferramenta. Dentre essas propriedades, é essencial testar a validade de construto que determina se o instrumento realmente mede o construto que se propõe a medir por meio de previsões com base na construção de hipóteses que são testadas para dar apoio à validade do instrumento. ${ }^{27}$

O FIT-HaNSA foi desenvolvido para avaliar a resistência durante tarefas que representam diferentes aspectos das funções motoras grossas do membro superior, como alcançar e pegar objetos em diferentes alturas e sustentar um trabalho acima da cabeça. ${ }^{28}$ Este instrumento tem sido muito utilizado na literatura para pacientes com lesões no ombro e cervical e encontramos estudos de validade apenas para esta população. $26,28,29$

Desta forma, o objetivo do presente estudo é analisar a validade de construto do FIT-HaNSA em trabalhadores com sintomas de dor, desconforto ou fadiga no membro superior, por meio da análise da associação entre a resistência à fadiga do segmento com os escores de força muscular isocinética do ombro e cotovelo e da força isométrica de preensão palmar, e também com a capacidade para o trabalho e a disfunção do membro superior. Espera-se encontrar de moderada a forte correlação entre o desempenho no teste do FIT-HaNSA e a força muscular, moderada correlação entre o FIT-HaNSA e a capacidade para o trabalho (ICT) e moderada correlação inversa entre o FIT-HaNSA e o Quick-DASH-Br.

\section{MÉTODO}

Trata-se de um estudo observacional transversal, aprovado pelo Comitê de Ética em Pesquisa da FMRP-USP (CAAE 89138818.1.0000.5440), em conformidade com a Declaração de Helsinki. Todos os voluntários concordaram em participar do estudo mediante assinatura do Termo de Consentimento Livre e Esclarecido (TCLE).

\section{Amostra}

Este estudo foi realizado com uma amostra de conveniência de 39 trabalhadores do HCFMRP-USP que foram recrutados por convite verbal. As coletas foram realizadas na sala de avaliação Isocinética do Centro de Reabilitação do Hospital das Clínicas da Faculdade de Medicina de Ribeirão Preto da Universidade de São Paulo (CER-HCFMRP-USP), com duração média de 40 minutos cada.

O estudo incluiu indivíduos com idade entre 18 e 60 anos, de ambos os gêneros, que tivessem tipo dor leve a moderada ou desconforto unilateral ou bilateral no membro superior nos últimos 12 meses. 
Nos casos de sintomas bilaterais, a lateralidade foi definida pelo membro superior que apresentava mais dor na Escala Numérica da dor (END). ${ }^{30}$ Indivíduos com histórico de trauma ou cirurgia recente ou incapazes de executar os testes propostos por motivos físicos ou cognitivos foram excluídos do estudo.

Teste FIT-HaNSA de Avaliação da resistência à fadiga do membro superior (versão brasileira)

Um protótipo (Figura 1) do equipamento JobSim ${ }^{\mathrm{TM}}$ System (JTECH Medical Industries, Inc. USA) (Figura 2) foi desenvolvido em 2016 e utilizado para a realização do FIT-HaNSA, cuja função é avaliar a resistência à fadiga e o desempenho funcional da extremidade superior durante tarefas que representem as funções motoras grossas da extremidade superior, tais como alcançar e segurar objetos em diferentes alturas e realizar um trabalho sustentado acima da cabeça. ${ }^{26}$ Este teste consiste em três tarefas que simulam atividades repetitivas de elevação do membro superior sustentada em diferentes níveis, associada a cargas.

Primeira tarefa - Waist-up: A primeira prateleira é colocada ao nível da cintura e a segunda $25 \mathrm{~cm}$ acima desta. Três recipientes pesando $1 \mathrm{~kg}$ cada são colocados na prateleira mais baixa. O participante é instruído a deslocar os três recipientes, de uma prateleira à outra com uma mão numa velocidade de 60 batidas/minuto controlada por um metrônomo.

Segunda tarefa - Eye-Down: Uma prateleira é colocada ao nível dos olhos e a outra $25 \mathrm{~cm}$ abaixo desta. O participante é novamente instruído a deslocar os três recipientes de uma prateleira a outra utilizando apenas uma mão em uma velocidade de 60 batidas/minuto.

Terceira tarefa - Overhead-Work: Uma prateleira é colocada ao nível dos olhos e nela é acoplada a placa de metal contendo parafusos. O participante é instruído a manter ambos os braços elevados e usá-los para enroscar e desenroscar os parafusos em uma sequência pré-determinada: o parafuso que está no nível 1 (topo) deve ser deslocado para o nível 2 (meio); o parafuso do nível 3 (inferior) para o nível 1 e o parafuso do nível 2 para o nível 3.

Entre cada tarefa são fornecidos 30 segundos de descanso, que é o tempo utilizado para reajustar as prateleiras.

Cada tarefa é realizada apenas uma vez por no máximo 300 segundos ou quando o voluntário utilizar os critérios de parada do teste, que são: realizar compensação com movimentos de tronco não corrigível em até cinco repetições sucessivas, interromper o teste por dor, fadiga ou incapacidade de completar uma repetição do movimento dentro de duas batidas do metrônomo por cinco repetições sucessivas.

\section{Dinamometria Isocinética de Avaliação da Força Muscular}

O teste de força muscular foi realizado utilizando o dinamômetro isocinético Biodex System 4 Pro $^{\mathrm{TM}}$, (Biodex Medical Systems, Inc., Shirley, NY, USA), de acordo com as recomendações de calibração e uso propostas pelo fabricante no manual.

Os movimentos avaliados pelo dinamômetro isocinético foram a abdução do ombro no plano da escápula e flexão do cotovelo (ICC $0,95-0,96) \cdot{ }^{31}$ A posição do dinamômetro para a
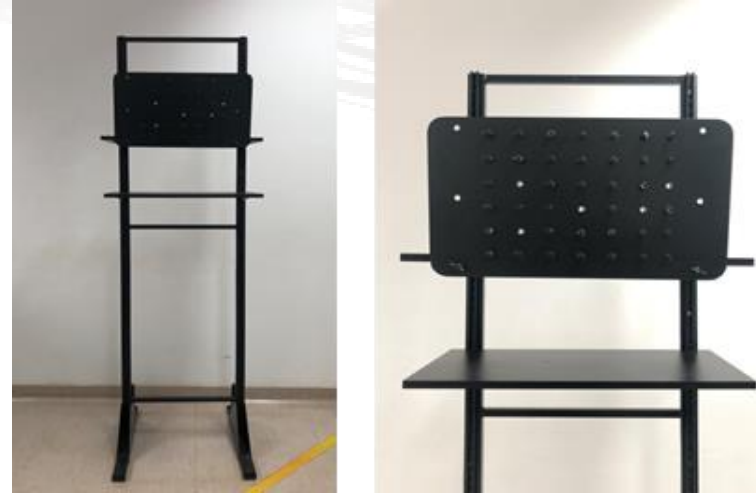

Figura 1. Versão brasileira do Teste FIT-HaNSA

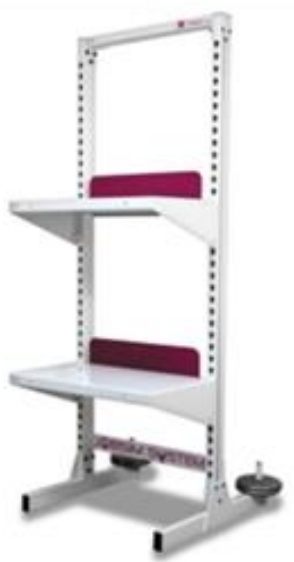

Figura 2. Equipamento JobSim ${ }^{\mathrm{TM}}$ System (JTECH Medical Industries, USA)

análise da flexão de cotovelo foi baseada no material de orientação de posicionamento disponibilizado pelo sistema Biodex $^{\mathrm{TM}}$ e o movimento de abdução do ombro foi adaptado para ser realizado no plano da escápula. O plano escapular foi baseado na angulação proposta por Kapandji, ${ }^{32}$ posicionando o ombro a 30 을 graus anteriormente ao plano frontal, mensurado por um goniômetro.

A variável de força utilizada para a análise das correlações foi a média do pico de torque, e baseado nas velocidades estudadas por Prentice \& Voight, ${ }^{33}$ todos os movimentos foram avaliados a uma velocidade de 60\%/seg, velocidade recomendada para avaliação de pessoas com queixas ortopédicas do membro superior. Portanto, avaliamos a média do pico de torque de abdução de ombro (MOABs) e de flexão do cotovelo (MCFs).

\section{Dinamometria de preensão palmar JAMAR ${ }^{\circledR}$}

O dinamômetro analógico da marca $\mathrm{JAMAR}^{\circledR}$ é um instrumento recomendado pela Sociedade Americana de Terapeutas de Mão (SATM) para medir a força de preensão palmar em quilogramas/força. ${ }^{34}$

Ele é considerado "padrão ouro" por apresentar dados normativos tanto para crianças quanto adultos ${ }^{35}$ e também por outros estudos já terem reportado a confiabilidade e validade deste instrumento. ${ }^{34} \mathrm{~A}$ avaliação da força de preensão é frequentemente usada no cenário clínico como um indicador da força física e da saúde em geral e não são vistas apenas 
como uma medida intrínseca da força das mãos ou mesmo limitadas à avaliação dos membros superiores. ${ }^{36}$

\section{Índice de Capacidade para o trabalho (ICT)}

O Índice de Capacidade para o Trabalho (ICT) é um instrumento que avalia por meio do autorrelato do trabalhador, a sua percepção sobre as condições de trabalho, capacidade física, mental e social que podem estar relacionadas às suas queixas, permitindo a avaliação de alterações importantes para esta população e a obtenção de informações que possam orientar medidas preventivas.

O índice contém dez questões divididas em sete domínios e apresentou boa confiabilidade pelo coeficiente alfa de Cronbach $(0,72)$. Os resultados fornecem uma medida de capacidade para o trabalho, que varia de 07 a 49 pontos, e classificam a capacidade em baixa (07 a 27), moderada (28 a 36), boa (37 a 43) e ótima (44 a 49). ${ }^{19}$

\section{Questionário de disfunção Quick-DASH-Br}

O Quick-DASH é um questionário de autorrelato, composto por 11 itens extraídos do DASH completo que avalia a disfunção e sintomas no membro superior percebidos pelo paciente na semana anterior.

Seus itens questionam sobre o grau de dificuldade para realizar várias atividades com a extremidade superior, qual a intensidade da dor e formigamento, bem como o impacto do problema na vida social, profissional e sono. Cada item é possui 5 opções de resposta que vai de 0 (nenhuma dificuldade/sintoma) a 5 (incapaz de realizar).

Sua pontuação final variando de 0 a 100, sendo a máxima pontuação indicativa de maior incapacidade no membro superior. ${ }^{18}$ Sua versão em português do Brasil (Quick-DASH-Br) foi validada para as disfunções do membro superior. ${ }^{18}$

\section{Escala Numérica de dor (END)}

A Escala Numérica de dor consiste numa régua numerada de zero a dez na qual o zero significa a ausência de dor e o dez uma dor inimaginável. Os participantes responderam a seguinte pergunta: “Qual é a intensidade de sua dor no momento, considerando zero ausência de dor e dez a pior dor possível?". Os valores são classificados como zero "sem dor", de 01 a 03 "dor leve", de 04 a 06 "dor moderada" e de 07 a 10 "dor intensa". ${ }^{30}$

\section{Questionário Nórdico de Sintomas Osteomusculares}

Com o objetivo de padronizar a mensuração do relato qualitativo da localização, característica e cronicidade dos sintomas, o questionário Nórdico de Sintomas Osteomusculares foi desenvolvido.

Consiste em um mapa corporal dividido em 09 segmentos, sendo eles: pescoço, ombros, parte superior das costas, cotovelos, punhos/mãos, parte inferior das costas, quadris/coxas, joelhos e tornozelos/pés.

Possui quatro questões de resposta binária sim/não para cada segmento a respeito dos distúrbios musculoesqueléticos, e demonstrou substancial concordância pelo coeficiente Kappa $(0.88-1))^{37}$

\section{Inventário de Preferência Lateral Global (IPLAG)}

Para avaliação da lateralidade foi aplicado o Inventário de Preferência Lateral Global (IPLAG) que objetiva analisar a preferência manual através de tarefas representativas das atividades de vida diária. O questionário é dividido em oito partes podendo ser utilizado em sua totalidade ou somente na dimensão requerida para análise. O participante responde as questões de acordo com sua preferência as opções: sempre esquerda, maioria esquerda, indiferente, maioria direita, sempre direita e não sei. Ao final, os pontos são somados para o cálculo da média geral e a classificação em destro, ambidestro ou canhoto é realizada. ${ }^{38}$

\section{Questionário Internacional de Atividade Física (IPAQ)- Versão Curta}

O nível de atividade física foi utilizado para critérios de caracterização da amostra e definido pelo Questionário Internacional de Atividade Física (IPAQ) - versão curta, que é um instrumento válido e confiável $($ ICC 0,77$)$ e permite estimar o tempo semanal de gasto energético em atividades físicas realizadas em uma semana normal, levando em consideração atividades de caminhada, atividades com intensidade moderada e vigorosa que tiveram duração mínima de 10 minutos contínuos, além do tempo despendido na posição sentada, sendo os participantes caracterizados em muito ativos, ativos e irregularmente ativos. ${ }^{39}$

\section{Procedimento para coleta de dados}

A Avaliação isocinética foi aplicada por fisioterapeutas previamente treinados. Um estudante de fisioterapia aplicou os questionários e o outro o teste FIT-HaNSA e a dinamometria com o $\mathrm{JAMAR}^{\circledR}$, sorteando a ordem de aplicação para cada voluntário. Inicialmente, os participantes forneceram seus dados por meio de um questionário sócio demográfico. Logo após, realizaram o primeiro teste sorteado, em seguida responderam o questionário Nórdico, a END, o IPLAG, o IPAQ e o Quick-DASH-Br para, finalmente, executar o segundo teste.

Os questionários foram feitos entre um teste e o outro para critérios de descanso e o teste foi iniciado com o membro superior sem queixa ou com menor dor determinada pela escala numérica de dor (END). Na avaliação de força com o $\mathrm{JAMAR}^{\circledR}$, foram coletadas 3 medidas de força de cada lado e calculada a média dessas medidas.

A validade do construto foi analisada pelo Coeficiente de Correlação de Pearson ( $r$ ) que varia de 0 a 1 . Os valores foram interpretados de acordo com a classificação de Di Fabio ${ }^{40}$ que considera uma forte correlação entre 0,75 e 1,0, correlação moderada entre 0,74 e 0,5, correlação fraca entre 0,49 e 0,25 e nenhuma ou pouca correlação se seu valor for igual a 0,24 , com valores de significância definidos em $p<0,05$ e intervalo de confiança de $95 \%$, pelo software SPSS ${ }^{\circledR} 20.0$.

\section{RESULTADOS}

Dos trinta e nove participantes do estudo, $61,5 \%$ eram do sexo feminino, com média de idade de 42,9 anos (DP 13,28). Todos os participantes utilizavam predominantemente os membros superiores em suas funções. 
Referente ao nível de atividade física analisado pelo IPAQ em 38 voluntários, 9 eram muito ativos, 11 ativos, 17 irregularmente ativos e apenas 1 sedentário.

Com relação à região acometida, 60,5\% dos trabalhadores sofriam de sintomas no ombro (destes, $69,5 \%$ ombro D); $23,7 \%$ sofriam de sintomas no cotovelo (66\% cotovelo D) e $15,8 \%$ percebiam sintomas no punho/mão ( $50 \%$ punho/mão D). Os demais dados de caracterização da amostra estão apresentados na Tabela 1.

\section{Avaliação da dor}

Vinte e sete dos trinta e nove indivíduos responderam o questionário Nórdico que identificou início dos sintomas no membro superior há pelo menos 12 meses em todos os casos. Dezenove participantes $(70,37 \%)$ queixaram-se dos mesmos sintomas nos últimos 07 dias.

O questionário também apontou que a predominância dos sintomas foi na articulação do ombro (15 participantes) seguido por cotovelo (7) e punho/mão (5), e que $88,88 \%$ da amostra referia dor em outros segmentos, como coluna torácica (17), cervical (15), lombar (13), joelho (10) tornozelo/pé (9) e quadril (4).

Com relação à intensidade dolorosa, toda a amostra avaliada $(n=27)$ relatou que a intensidade da dor oscilava dependendo do nível de esforço empregado no membro ou da postura adotada ao longo de um dia, com piora dos sintomas durante a jornada de trabalho em $77,77 \%$ dos respondentes. Esta mesma porcentagem de participantes relatou estar com dor no momento da avaliação, classificada como "leve" pela END.

A END foi reaplicada no final da avaliação em 18 participantes, dos quais $50 \%$ mantiveram a intensidade inicial, $38,88 \%$ referiram aumento da dor e $11,11 \%$ diminuição da dor.

Tabela 1. Descrição demográfica dos participantes

\begin{tabular}{ll}
\hline \multicolumn{1}{c}{ Características } & \multicolumn{1}{c}{$\mathbf{N}=\mathbf{3 9}$} \\
\hline Idade & $42,9 \pm 13,28$ \\
Gênero (feminino/masculino) & $24 / 15$ \\
& Normal 38,4\% \\
IMC (\%) & Sobrepeso 25,6\% \\
& Obesidade 36\% \\
& 29 destros/ \\
Dominância (IPLAG) & 2 canhotos/ \\
& 1 ambidestro*
\end{tabular}

IMC: Índice de Massa Corporal; IPLAG: Inventário de Preferência Lateral Global; ${ }^{*} N=33,6$ não identificado

\section{Validade de Construto}

Durante as coletas, tivemos algumas perdas na aplicação de alguns instrumentos. A correlação entre o FIT-HaNSA e o JAMAR, ICT e QuickDASH-Br foi conduzida com 29, 27 e 39 voluntários da amostra total, respectivamente. Para correlacionar o FIT-HaNSA com o limiar de dor à pressão, com a MOABs e com a MCFs, utilizamos os dados de 37, 26 e 26 voluntários do total, respectivamente.

O FIT-HaNSA apresentou fraca correlação inversa com a pontuação total do QuickDASH-Br $(r=-0,38, p=0,16)$ e pouca correlação com a pontuação no ICT $(r=0,21, p=0,28)$. A análise entre o FIT-HaNSA e o grau de força de preensão manual obtido no JAMAR apresentou moderada correlação significativa ( $r=$ $0,58, p=0,001$ ); entre o FIT-HaNSA e a MOABs foi observada correlação fraca, porém significativa $(r=0,43, p=0,028)$ e quando comparado à MCFs pouca correlação foi estabelecida $(r=0,18, p=0,36)$.

Nas demais análises de correlação realizadas entre as variáveis, encontramos forte correlação apenas na associação da MOABs com o JAMAR ( $r=0,72, p<0,001, n=26)$ e com a MCFs $(r=0,76, p<0,001, n=25)$. As demais correlações estão apresentadas na Tabela 2. As médias dos escores das ferramentas, estão descritas na Tabela 3.

Tabela 2. Matriz de correlações de Pearson ( $r$ ) entre os Instrumentos de Medida

\begin{tabular}{|c|c|c|c|c|c|}
\hline & ICT & JAMAR & MOABs & MCFs & QuickDASH-Br \\
\hline \multirow[t]{3}{*}{ QuickDASH-Br } & $r=-0,60 *$ & $r=-0,51^{*}$ & $r=-0,33$ & $r=-0,37$ & $r=1$ \\
\hline & $p=0,001$ & $p=0,004$ & $p=0,09$ & $p=0,06$ & \\
\hline & $\mathrm{N}=27$ & $\mathrm{~N}=29$ & $\mathrm{~N}=26$ & $\mathrm{~N}=26$ & $N=39$ \\
\hline \multirow[t]{3}{*}{ ICT } & $r=1$ & $r=0,26$ & $r=0,20$ & $r=0,32$ & $r=-0,60 *$ \\
\hline & & $p=0,16$ & $p=0,31$ & $p=0,10$ & $p=0,001$ \\
\hline & $N=27$ & $N=27$ & $N=26$ & $N=26$ & $N=27$ \\
\hline \multirow[t]{3}{*}{ JAMAR } & $r=0,26$ & $r=1$ & $r=0,72 *$ & $r=0,33$ & $r=-0,51^{*}$ \\
\hline & $p=0,16$ & & $p=0,000$ & $p=0,09$ & $p=0,004$ \\
\hline & $N=27$ & $N=29$ & $N=26$ & $N=26$ & $N=29$ \\
\hline \multirow[t]{3}{*}{ MOABs } & $r=0,20$ & $r=0,72^{*}$ & $r=1$ & $r=0,76^{*}$ & $r=-0,33$ \\
\hline & $p=0,31$ & $p=0,000$ & & $p=0,000$ & $p=0,09$ \\
\hline & $N=26$ & $N=26$ & $N=26$ & $\mathrm{~N}=25$ & $N=26$ \\
\hline \multirow[t]{3}{*}{ MCFs } & $r=0,32$ & $r=0,33$ & $r=0,76^{*}$ & $r=1$ & $r=-0,37$ \\
\hline & $p=0,10$ & $p=0,09$ & $p=0,000$ & & $p=0,06$ \\
\hline & $N=26$ & $N=26$ & $N=25$ & $N=26$ & $N=26$ \\
\hline
\end{tabular}

ICT: Índice de Capacidade para o Trabalho; JAMAR: Dinamômetro de Preensão JAMAR; MCFs: Média do pico de torque para flexão de cotovelo; MOABs: Média do pico de torque para abdução do ombro no plano escapula; $N=$ Amostra; $p=$ Nível de significância; $r=$ Coeficiente de Correlação de Pearson

Tabela 3. Score dos Instrumentos de Medida

\begin{tabular}{lccc}
\hline \multicolumn{1}{c}{ Instrumento } & $\mathbf{n}$ & Média & DP \\
\hline FIT-HaNSA - FMT & 39 & $186,13 \mathrm{~s}$ & 71,02 \\
QuickDASH-Br & 39 & 26,97 & 19,65 \\
ICT & 27 & 36,3 & 5,54 \\
JAMAR $\AA$ & 20 & D 28,21 / E 26,93 & $11,02 / 11,23$
\end{tabular}

D: Membro superior direito; DP: Desvio Padrão; E: Membro superior esquerdo; ICT: Índice de Capacidade para o Trabalho; FMT: Média dos scores das 3 tarefas do FITHaNSA; JAMAR: Dinamômetro de preensão JAMAR ${ }^{\oplus}$; QuickDASH-Br: Quick Disabilities of the Arm, Shoulder and Hand Versão Brasileira

\section{DISCUSSÃO}

Até o momento, é escasso o número de estudos que utilizaram o FIT-HaNSA para a avaliação da fadiga em 
trabalhadores e encontramos na literatura apenas dados de validação desta ferramenta para disfunção no ombro. ${ }^{26,28,29}$

Os achados do presente estudo demonstraram de pouca a moderada associação do FIT-HaNSA com o grau de força muscular, e de pouca a fraca associação com incapacidade para o trabalho e disfunção no membro superior, sugerindo que ele mede um diferente construto.

Concernente às hipóteses pré estabelecidas em nosso estudo, apenas a correlação moderada entre o FIT-HaNSA e a força de preensão no $\operatorname{JAMAR}^{\circledR}$ foi confirmada, já que a associação com a força de abdução do ombro foi fraca, apesar de significativa, e pouca correlação foi estabelecida com a força de flexão de cotovelo. Isto pode ser explicado pela diferença de propriedade muscular exigida, já que as tarefas do FIT-HaNSA estão mais relacionadas à resistência muscular do que à força.

Nossa hipótese de moderada correlação entre o FIT-HaNSA e os questionários de autorrelato ICT e QuickDASH-Br também foi refutada, o que pode ser explicado pelo fato da autopercepção do paciente nem sempre refletir a realidade avaliada pelos profissionais, por ser influenciada por fatores pessoais, ambientais, depressão, educação, comprometimento cognitivo, entre outros. ${ }^{28}$

Nosso estudo também divergiu da média de tempo para a realização das tarefas 1, 2 e 3 do FIT-HaNSA. Enquanto nossa amostra obteve uma média de respectivamente, 233, 143 e 188 segundos, o estudo que desenvolveu a ferramenta bem como um estudo recente que validou a mesma para o ombro apresentaram para os pacientes sintomáticos médias menores de tempo de respectivamente, 179, 117 e 151 segundos $^{26}$ e 201, 117 e 170 segundos $^{29}$ em cada uma das 3 tarefas. Provavelmente esta diferença seja explicada pela diferença da nossa amostra com queixas dolorosas com a população desses estudos, que apresentavam disfunção somente no ombro e com diagnóstico de lesão já estabelecido.

Entre as 3 tarefas do FIT-HaNSA, a tarefa 2 foi a que apresentou os piores resultados em nosso estudo, o que também foi observado por outros estudos que utilizaram esta ferramenta. ${ }^{26,28,29,41} \mathrm{~A}$ tarefa 2 foi a tarefa que ofereceu maior dificuldade por exigir movimentos repetidos em uma posição de relativo impacto do ombro durante uma tarefa de preensão e alcance, além da extremidade superior já estar "pré-fatigada" pela tarefa $1 .{ }^{28,29}$

No que diz respeito à correlação com as medidas de dor e força, nossos resultados concordam com os observados em estudos de validação para ombro. ${ }^{26,28,29}$ O questionário Nórdico classificou que nossos pacientes possuíam dor crônica, com presença de sintomas em mais de um segmento. A dor crônica entre população em idade laboral vem alcançando proporções cada vez maiores no Brasil, tornando-se uma preocupação prioritária para os sistemas de saúde por gerar impactos negativos aos empregadores e à economia. ${ }^{42}$

Com relação à associação entre o FIT-HaNSA e o DASH, questionário equivalente e que originou o Quick-DASH-Br, dois estudos preliminares encontraram forte associação inversa entre os instrumentos $(r=-0,94$ e $r=-0,76),{ }^{26,28}$ divergindo dos nossos achados. Porém, Pierrynowski ${ }^{29}$ e Roy et al. ${ }^{41}$ também encontraram fraca associação do FIT-HaNSA com o DASH ( $r=-$ 0,34 e $r=-0,31$, respectivamente). Os escores do Quick-DASH e ICT da nossa população de trabalhadores os classificou com baixo nível de incapacidade do membro superior e de moderada a boa capacidade para o trabalho. Além disso, encontrou significativa correlação inversa entre a incapacidade e a força de preensão manual, evidenciando que a incapacidade do membro superior diminuiu quando a força de preensão aumentou. Este dado concorda com Novaes et al. ${ }^{43}$ que afirma força de preensão manual está fortemente relacionada com o bom estado geral de saúde.

De acordo com Pierrynowski ${ }^{29}$ a relação entre desempenho físico, dor, capacidade e comprometimento, sejam eles determinados pela autopercepção ou desempenho real, são variadas e complexas, mas estas medidas devem ser juntamente utilizadas por trazer informações sobre diferentes aspectos da saúde humana na presença de disfunções.

\section{Limitações do estudo}

Nossa metodologia e o tamanho da amostra para as análises conduzidas não permitiu estabelecer um nexo causal entre as variáveis analisadas os sintomas percebidos pelos trabalhadores, além de não ter sido realizada uma avaliação mais minuciosa das características ergonômicas de trabalho.

Além disso, não aplicamos nenhum instrumento que avaliasse as condições psicossociais que os trabalhadores se encontravam, sabendo que a capacidade para o trabalho também é influenciada pelos aspectos psicológico, cognitivo e social, que sofrem interferência da dor, diminuindo a habilidade para trabalhar. ${ }^{44}$

Conhecer de forma abrangente os fatores de risco para desenvolvimento de queixas musculoesqueléticas relacionadas ao trabalho através de uma avaliação padronizada, com o objetivo de estabelecer intervenções eficazes e estratégias de prevenção se faz necessário. Uma vez que a capacidade física está relacionada à ocorrência de sintomas musculoesqueléticos nos trabalhadores, quando consideradas características individuais e ocupacionais. ${ }^{45}$

Novos estudos prospectivos são necessários para a busca de evidências sobre métodos de avaliação válidos, confiáveis e responsivos acerca das queixas e disfunções musculoesqueléticas.

\section{CONCLUSÃO}

O FIT-HaNSA mostrou ser válido para avaliação da resistência muscular à fadiga na população estudada.

Nossas hipóteses de correlação foram parcialmente confirmadas, tal ferramenta correlacionou-se significativamente com a força de preensão manual e com a média do pico de força de abdução do ombro. Nosso estudo forneceu evidências preliminares com relação às relações esperadas e validade de construto do FIT-HaNSA com os instrumentos de medida selecionados para a avaliação de trabalhadores com queixas no membro superior.

\section{AGRADECIMENTOS}

Agradecemos a todos os pesquisadores envolvidos no desenvolvimento do estudo e principalmente aos fisioterapeutas do Centro de Reabilitação do Hospital das Clínicas de Ribeirão Preto que apoiaram a coleta de dados. 


\section{REFERENCIAS}

1. Soyer K, Unver B, Tamer S, Ulger O. The importance of rehabilitation concerning upper extremity amputees: a Systematic review. Pak J Med Sci. 2016;32(5):1312-9. Doi: https://doi.org/10.12669/pjms.325.9922

2. Schuind FA, Mouraux D, Robert C, Brassinne E, Rémy P, Salvia $P$, et al. Functional and outcome evaluation of the hand and wrist. Hand Clin. 2003;19(3):361-9. Doi: https://doi.org/10.1016/S0749-0712(03)00026-X

3. Van der Heide LA, van Ninhuijs B, Bergsma A, Gelderblom GJ, van der Pijl DJ, de Witte LP. An overview and categorization of dynamic arm supports for people with decreased arm function. Prosthet Orthot Int. 2014;38(4):287-302. Doi: https://doi.org/10.1177/0309364613498538

4. Roshanravan B, Patel KV, Fried LF, Robinson-Cohen C, de Boer $\mathrm{IH}$, Harris $\mathrm{T}$, et al. Association of muscle endurance, fatigability, and strength with functional limitation and mortality in the health aging and body composition study. J Gerontol A Biol Sci Med Sci. 2017;72(2):284-291. Doi: https://doi.org/10.1093/gerona/glw210

5. Van Harlinger W, Blalock L, Merritt JL. Upper limb strength: study providing normative data for a clinical handheld dynamometer. PM R. 2015;7(2):135-40. Doi: https://doi.org/10.1016/j.pmrj.2014.09.007

6. Nordander C, Ohlsson K, Akesson I, Arvidsson I, Balogh I, Hansson GA, et al. Risk of musculoskeletal disorders among females and males in repetitive/constrained work. Ergonomics. 2009;52(10):1226-39. Doi: https://doi.org/10.1080/00140130903056071

7. Sundstrup $\mathrm{E}$, Jakobsen $\mathrm{MD}$, Andersen $\mathrm{CH}$, Jay $\mathrm{K}$, Persson $\mathrm{R}$, Aagaard $\mathrm{P}$, et al. Effect of two contrasting interventions on upper limb chronic pain and disability: a randomized controlled trial. Pain Physician. 2014;17(2):145-54.

8. Thacham Poyil A, Steuber V, Amirabdollahian F. Adaptive robot mediated upper limb training using electromyogrambased muscle fatigue indicators. PLoS One. 2020;15(5):e0233545.

https://doi.org/10.1371/journal.pone.0233545

Doi:

9. Johansen $H$, Bathen $T$, Andersen $L \varnothing$, Rand-Hendriksen $S$, $\emptyset$ stlie K. Chronic pain and fatigue in adults with congenital unilateral upper limb deficiency in Norway. a cross-sectional study. PLoS One. 2018;13(1):e0190567. Doi: https://doi.org/10.1371/journal.pone.0190567

10. Van Eerd D, Munhall C, Irvin E, Rempel D, Brewer S, van der Beek AJ, et al. Effectiveness of workplace interventions in the prevention of upper extremity musculoskeletal disorders and symptoms: an update of the evidence. Occup Environ Med. 2016;73(1):62-70. Doi: https://doi.org/10.1136/oemed2015-102992

11. Lomond KV, Côté JN. Shoulder functional assessments in persons with chronic neck/shoulder pain and healthy subjects: reliability and effects of movement repetition. Work. 2011;38(2):169-80.

Doi https://doi.org/10.3233/WOR-2011-1119

12. Jacobs NW, Berduszek RJ, Dijkstra PU, van der Sluis CK. Validity and Reliability of the Upper Extremity Work Demands Scale. J Occup Rehabil. 2017;27(4):520-529. Doi: https://doi.org/10.1007/s10926-016-9683-9

13. Skirven TM, Osterman AL, Fedorczyk J, Amadio PC. Rehabilitation of the hand and upper extremity. 6th ed. St. Louis: Mosby; 2011.
14. Macdermid JC. The Patient Rated Wrist Evaluation (PRWE) user manual. Ontario: School of Rehabilitation Science, McMaster University; 2011.

15. Beattie P. Measurement of health outcomes in the clinical setting: applications to physiotherapy. Physiother Theory Pract. 2001;17(3):173-85. Doi: https://doi.org/10.1080/095939801317077632

16. Walker LAS, Lindsay-Brown AP, Berard JA. Cognitive Fatigability Interventions in Neurological Conditions: A Systematic Review. Neurol Ther. 2019;8(2):251-271. Doi: https://doi.org/10.1007/s40120-019-00158-3

17. Orfale AG, Araújo PM, Ferraz MB, Natour J. Translation into Brazilian Portuguese, cultural adaptation and evaluation of the reliability of the Disabilities of the Arm, Shoulder and Hand Questionnaire. Braz J Med Biol Res. 2005;38(2):293302. Doi: https://doi.org/10.1590/s0100879x2005000200018

18. Silva NC, Chaves TC, Santos JB, Sugano RMM, Barbosa RI, Marcolino AM, et al. Reliability, validity and responsiveness of Brazilian version of QuickDASH. Musculoskelet Sci Pract. 2020;48:102163.

Doi: https://doi.org/10.1016/j.msksp.2020.102163

19. Martinez MC, Latorre MRDO, Fischer FM. Validade e confiabilidade da versão brasileira do Índice de Capacidade para o Trabalho. Rev Saúde Pública. 2009;43(3):525-32. Doi: http://dx.doi.org/10.1590/S0034-89102009000300017

20. Gabel CP, Michener LA, Burkett B, Neller A. The Upper Limb Functional Index: development and determination of reliability, validity, and responsiveness. J Hand Ther. 2006;19(3):328-48.

Doi: http://dx.doi.org/10.1197/j.jht.2006.04.001

21. Chung KC, Pillsbury MS, Walters MR, Hayward RA. Reliability and validity testing of the Michigan Hand Outcomes Questionnaire. J Hand Surg Am. 1998;23(4):575-87. Doi: http://dx.doi.org/10.1016/S0363-5023(98)80042-7

22. Opsteegh L, Soer R, Reinders-Messelink HA, Reneman MF, van der Sluis CK. Validity of the dictionary of occupational titles for assessing upper extremity work demands. PLoS One. 2010;5(12):e15158.

Doi: http://dx.doi.org/10.1371/journal.pone.0015158

23. Gouveia VV, Oliveira GF, Mendes LAC, Souza LEC, Cavalcanti TM, Melo RLP. Escala de avaliação da fadiga: adaptação para profissionais da saúde. Rev Psi Organ Trab. 2015;15(3):24656. Doi: http://dx.doi.org/10.17652/rpot/2015.3.594

24. Gorman PP, Butler RJ, Plisky PJ, Kiesel KB. Upper Quarter Y Balance Test: reliability and performance comparison between genders in active adults. J Strength Cond Res. 2012;26(11):3043-8.

Doi: http://dx.doi.org/10.1519/JSC.0b013e3182472fdb

25. Salo TD, Chaconas E. The Effect of fatigue on upper quarter $y$ balance test scores in recreational weightlifters: a randomized controlled trial. Int J Sports Phys Ther. 2017;12(2):199-205.

26. MacDermid JC, Ghobrial M, Quirion KB, St-Amour M, Tsui T, Humphreys $D$, et al. Validation of a new test that assesses functional performance of the upper extremity and neck (FITHaNSA) in patients with shoulder pathology. BMC Musculoskelet Disord. 2007;8:42. Doi: http://dx.doi.org/10.1186/1471-2474-8-42 
27. Mokkink LB, Terwee CB, Patrick DL, Alonso J, Stratford PW, $\mathrm{Knol} \mathrm{DL}$, et al. The COSMIN study reached international consensus on taxonomy, terminology, and definitions of measurement properties for health-related patient-reported outcomes. J Clin Epidemiol. 2010;63(7):737-45. Doi: http://dx.doi.org/10.1016/j.jclinepi.2010.02.006

28. Kumta P, MacDermid JC, Mehta SP, Stratford PW. The FITHaNSA demonstrates reliability and convergent validity of functional performance in patients with shoulder disorders. J Orthop Sports Phys Ther. 2012;42(5):455-64. Doi: http://dx.doi.org/10.2519/jospt.2012.3796

29. Pierrynowski M, McPhee C, P Mehta S, C MacDermid J, Gross A. Intra and inter-rater reliability and convergent validity of FIT-HaNSA in individuals with grade $\Pi$ whiplash associated disorder. Open Orthop J. 2016;10:179-89. Doi: https://doi.org/10.2174/1874325001610010179

30. Hartrick CT, Kovan JP, Shapiro S. The numeric rating scale for clinical pain measurement: a ratio measure? Pain Pract. 2003;3(4):310-6. Doi: $\quad$ https://doi.org/10.1111/j.15307085.2003.03034.x

31. Feiring DC, Ellenbecker TS, Derscheid GL. Test-retest reliability of the biodex isokinetic dynamometer. J Orthop Sports Phys Ther. 1990;11(7):298-300. Doi: https://doi.org/10.2519/jospt.1990.11.7.298

32. Kapandji Al. Fisiologia articular - membros superiores. 5 ed. Rio de Janeiro: Guanabara Koogan; 2000.

33. Prentice WE. Técnicas em reabilitação musculoesquelética. 4 ed. São Paulo: Artmed; 2003.

34. Reis MM, Arantes PMM. Medida da força de preensão manual- validade e confiabilidade do dinamômetro saehan. Fisioter Pesq. 2011;18(2):176-81. Doi: https://doi.org/10.1590/S1809-29502011000200013

35. Mathiowetz V. Comparison of Rolyan and Jamar dynamometers for measuring grip strength. Occup Ther Int. 2002;9(3):201-9. Doi: https://doi.org/10.1002/oti.165

36. Amaral JF, Mancini M, Novo Júnior JM. Comparison of three hand dynamometers in relation to the accuracy and precision of the measurements. Rev Bras Fisioter. 2012;16(3):216-24. Doi: https://doi.org/10.1590/s1413-35552012000300007
37. Pinheiro FA, Troccoli BT, Carvalho CV. Validação do Questionário Nórdico de Sintomas Osteomusculares como medida de morbidade. Rev Saúde Públ. 2002;36(3):307-12. Doi: https://doi.org/10.1590/S0034-89102002000300008

38. Marim EA, Lafasse R, Okazaki VHA. Inventário de preferência lateral global (IPLAG). Braz J Mot Behav. 2011; 6(3): 14-23. Doi: https://doi.org/10.13140/RG.2.1.1455.2163

39. Matsudo S, Araújo T, Matsudo V, Andrade D, Andrade E, Oliveira LC, et al. Questionário internacional de atividade fisica (IPAQ): estudo de validade e reprodutibilidade no Brasil. Rev Bras Ativ Fis Saúde. 2001;6(2):1-14. Doi: https://doi.org/10.12820/rbafs.v.6n2p5-18

40. Di Fabio RP. Essentials of rehabilitation research: a statistical guide to clinical practice. Philadelphia: F. A Davis; 2013.

41. Roy JS, Macdermid JC, Boyd KU, Faber KJ, Drosdowech D, Athwal GS. Rotational strength, range of motion, and function in people with unaffected shoulders from various stages of life. Sports Med Arthrosc Rehabil Ther Technol. 2009;1:4. Doi: https://doi.org/10.1186/1758-2555-1-4

42. Barreto IG, Sá KN. Prevalence and factors associated with chronic neuropathic pain in workers of a Brazilian public university. $\mathrm{Br} J$ Pain. 2019;2(2):105-11. Doi: https://doi.org/10.5935/2595-0118.20190020

43. Novaes RD, Miranda AS, Silva JO, Tavares BVF, Dourado VZ. Equações de referência para a predição da força de preensão manual em brasileiros de meia idade e idosos. Fisioter Pesq. 2009;16(3):217-22. Doi: https://doi.org/10.1590/S1809$\underline{29502009000300005}$

44. Hengstebeck E, Roskos S, Breejen K, Arnetz B, Arnetz J. Chronic pain disrupts ability to work by interfering with social function: A cross-sectional study. Scand J Pain. 2017;17:397402. Doi: https://doi.org/10.1016/j.sjpain.2017.09.021

45. Cabral AM, Moreira RFC, de Barros FC, Sato TO. Is physical capacity associated with the occurrence of musculoskeletal symptoms among office workers? A cross-sectional study. Int Arch Occup Environ Health. 2019;92(8):1159-1172. Doi: https://doi.org/10.1007/s00420-019-01455-y 